DRAFT VERSION JUNE 11, 2021

Preprint typeset using $\mathrm{LT}_{\mathrm{E}} \mathrm{X}$ style emulateapj v. 6/22/04

\title{
AN EFFICIENT METHOD TO IDENTIFY GALAXY CLUSTERS BY USING SUPERCOSMOS, 2MASS AND WISE DATA
}

\author{
W. W. XU ${ }^{1,2}$, Z. L. WEN ${ }^{1}$ AND J. L. HAN ${ }^{1}$ \\ Draft version June 11, 2021
}

\begin{abstract}
The survey data of Wide-field Infrared Survey Explorer (WISE) provide an opportunity for the identification of galaxy clusters. We present an efficient method for detecting galaxy clusters by combining the WISE data with SuperCOSMOS and 2MASS data. After performing star-galaxy separation, we calculate the number of companion galaxies around the galaxies with photometric redshifts previously estimated by the SuperCOSMOS, 2MASS and WISE data. A scaled richness is then defined to identify clusters. From a sky area of 275 $\operatorname{deg}^{2}$ coincident with Sloan Digital Sky Survey Stripe 82 region, we identify 302 clusters in the redshift range of $0.1<z<0.35,247$ (82\%) of which are previously known SDSS clusters. The results indicate that our method is efficient for identifying galaxy clusters by using the all sky data of the SuperCOSMOS, 2MASS and WISE.

Subject headings: Infrared; Galaxy clusters; Redshifts
\end{abstract}

\section{INTRODUCTION}

Galaxy clusters are known as the largest gravitational bounded systems in the universe. They are located at nods of cosmic web. The space distribution of galaxy clusters traces the large scale structure (Bahcall 1988; Allen et al. 2011; Hong et al. 2012). Their mass distribution can be used to constrain cosmological parameters (Reiprich \& Bohringer 2002; Wen et al. 2010). Clusters are also regarded as natural gravitational lens to magnify faint background sources (Wen et al. 2011; Liang et al. 2013) and laboratories to study galaxy evolution (Wen \& Han|2011; Liu et al. 2012). Discovery of galaxy clusters is the basis for many related studies.

In optical/infrared, a lot of methods have been used to detect clusters from image data. By visual inspection of optical images, Abell identified more than 4000 nearby rich clusters covering the whole sky (Abell 1958; Abell et al. 1989). Similar visual inspections were undertaken by Zwicky et al. (1968) and Gunn et al. (1986). For reducing subjectivity, automated peak-finding methods were developed, e.g., matchedfilter algorithm (Postman et al. 1996), adaptive kernel technique (Gal et al. 2003) and Voronoi tessellation techniques (Ramella et al. 2001; Kim et al. 2002). The single-band image data always suffer severe contamination from foreground and background galaxies and provide very poor redshift estimation of clusters.

The Sloan Digital Sky Survey (SDSS, York et al. 2000) offers an opportunity to identify a large number of clusters. It provides photometry in five broad bands $(u, g, r, i$, and $z)$ covering $\sim 14,000 \mathrm{deg}^{2}$, as well as the follow-up spectroscopic observations. In such multi-band surveys, galaxy colors are related to their redshifts. Member galaxies have similar colors and show a red sequence, so that proper cuts in colors can reduce projection effect for cluster identification. A lot of red-sequence based methods have been developed (Gladders \& Yee 2000, 2005; Goto et al. 2002; Koester et al. 2007). Photometric redshifts were estimated for all galaxies (Csabai et al. 2003; Oyaizu et al. 2008). A large number of galaxy clusters have been found based on photometric redshift (Wen et al. 2009, 2012; Szabo et al. 2011).

\footnotetext{
${ }^{1}$ National Astronomical Observatories, Chinese Academy of Sciences, Beijing 100012, China; zhonglue@ nao.cas.cn.

${ }^{2}$ University of Chinese Academy of Sciences, Beijing 100012, China
}

Except for Abell clusters at low redshift (Abell et al. 1989), there is no all sky cluster catalog up to intermediate redshift of $z \sim 0.3$. The Wide-field Infrared Survey Explorer (WISE) is an all-sky survey at infrared wavelengthes with the observation depth similar to that of the SDSS (Yan et al. 2013), which provides an opportunity to identify many new clusters covering the whole sky. Combined with optical data, the WISE data have been used to identify clusters at high redshift (Gettings et al. 2012).

In this paper, we present a simple but very efficient method to identify galaxy clusters by combing the WISE data with the SuperCOSMOS (Hambly et al. 2001) and Two Micron All Sky Survey (2MASS, Skrutskie et al. 2006) data. In Section 2 , we describe the data, present our method for the identification of galaxy clusters, and apply it to the data in the SDSS Stripe 82 region $^{3}$. In Section 3, we compare the identified clusters with previous SDSS clusters. A summary is given in Section 4.

\section{DATA AND ALGORITHM FOR CLUSTER IDENTIFICATION}

2MASS is an all-sky survey in three infrared bands, $J(1.25$ $\mu \mathrm{m}), H(1.65 \mu \mathrm{m})$ and $K_{s}(2.17 \mu \mathrm{m})$. The effective resolution of 2MASS is $\sim 5^{\prime \prime}$. The magnitude limits of 2MASS are $15.8,15.1$ and $14.3(10 \sigma)$ for point sources, and 15.0, 14.3 and 13.5 for extended sources in the three bands, respectively (Skrutskie et al. 2006).

The WISE observes the whole sky in four infrared bands, $W 1(3.4 \mu \mathrm{m}), W 2(4.6 \mu \mathrm{m}), W 3(12 \mu \mathrm{m})$ and $W 4(22 \mu \mathrm{m})$. The angular resolutions in the four bands are $6.1^{\prime \prime}, 6.4^{\prime \prime}, 6.5^{\prime \prime}$ and $12.0^{\prime \prime}$, respectively. The all sky survey depths are 16.5, 15.5, 11.2 and $7.9 \mathrm{mag}(5 \sigma)$ for point sources (Wright et al. 2010). The most important data for cluster identification are WISE $W 1$-band and 2MASS $J$-band magnitudes. In terms of detecting distant galaxies, the WISE data is much deeper than the 2MASS data. The limit of cluster detection mainly depends on the depth of the 2MASS data.

Because of the poor resolutions, both WISE and 2MASS data have no reliable star-galaxy separation for most objects by morphological parameters. However, stars and galaxies can be well separated by color index, $W 1-J$ (Kovács \& Szapudi 2013). We cross-match the 2MASS-

\footnotetext{
${ }^{3}$ http://cas.sdss.org/stripe82/en/
} 
WISE objects with known stars and galaxies in SDSS DR7 (Abazaiian et al. 2009) brighter than $r=21$ mag with a matching radius of $3^{\prime \prime}$. In Figure 2, we show the efficiency for the star-galaxy separation with the $W 1-J$ color index of the WISE-2MASS data. Galaxies are very well separated with the criteria of $W 1-J<-1.5 \mathrm{mag}$.

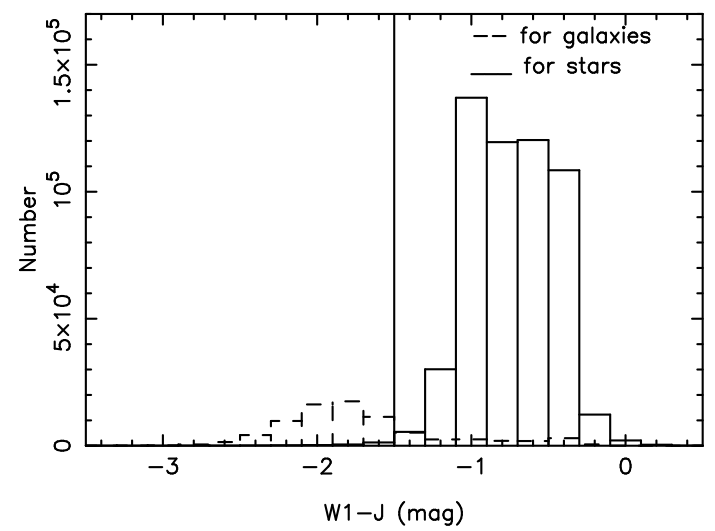

Figure 1 Separation of stars and galaxies by color index, $W 1-J$. The vertical line is $W 1-J=-1.5 \mathrm{mag}$, which is used as the threshold for star-galaxy separation.

To get redshift information for galaxy clusters, we use the catalog of galaxy photometric redshifts ${ }^{4}$ given by Bilicki et al. (2014) for 2MASS galaxies. By combining the data of SuperCOSMOS, 2MASS and WISE, these authors applied an artificial neural network approach to estimate photometric redshifts of galaxies covering the whole sky. The photometric redshift has an uncertainty of $\sigma_{z}=0.015$ and small percentage of outliers. Note that only 2MASS extended sources have photometric redshifts. The number of galaxies with photometric redshifts is about one tenth of the galaxies separated by $W 1-J<-1.5$.

Using the WISE-2MASS data together with photometric redshifts by Bilicki et al. (2014) in the SDSS Stripe 82 region of $309^{\circ} \leq \mathrm{RA} \leq 60^{\circ}$ and $-1.25^{\circ} \leq \mathrm{Dec} \leq 1.25^{\circ}$, we make cluster identification in the following steps.

For each galaxy with a photometric redshift, we take it as the temporary central galaxy of a cluster candidate, and the photometric redshift is taken as the redshift of the cluster candidate. We then calculate the number of companion galaxies from all separated galaxies within a projected distance of $1 \mathrm{Mpc}$. The average number of background galaxies is estimated using the galaxies within the projected distance between 2 and $4 \mathrm{Mpc}$ from the assumed central galaxy. We then get the net number of companion galaxies within $1 \mathrm{Mpc}$ of the central galaxy after background subtraction, which is taken as a measured richness, $R_{\text {mea }}$. To avoid a cluster identified repeatedly, we applied the friend-of-friend technique (Huchra \& Geller 1982) to merge the members into a cluster candidate, and consider only one cluster candidate within a projected distance of $1 \mathrm{Mpc}$ and a photometric redshift difference of 0.05 . We take the cluster candidate with the maximum measured richness, $R_{\text {mea }}$.

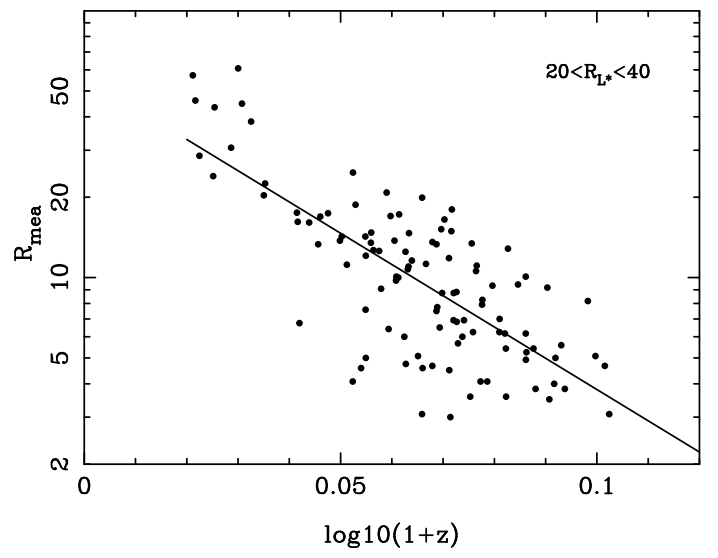

Figure 2 The value of $R_{\text {mea }}$ as a function of redshift for the matched WHL12 clusters of $20<R_{L *}<40$. The solid line is the best-fit relation.

The second step is to find a richness threshold to identify real galaxy clusters. The best selection is that the cluster richness is related to cluster mass. Because we use the fluxlimited galaxy sample of the WISE-2MASS data, the measured richness, $R_{\text {mea }}$, strongly depends on redshift for clusters with a fixed mass. Here, we define a scaled richness with redshift correction,

$$
R_{\text {scal }}=R_{\text {mea }}(1+z)^{\alpha},
$$

to relate cluster mass, where $\alpha$ is the correction slope. Note that the richness, $R_{L *}$, in the catalog of Wen et al. (2012, WHL12 hereafter) has a good correlation with cluster mass. Thus, we cross-match the identified cluster candidates with the WHL12 clusters of $20<R_{L *}<40$ by using criteria of a projected separation of $1 \mathrm{Mpc}$ and a redshift difference of 0.05 . To get a proper value of $\alpha$, we plot $R_{\text {scal }}$ as a function of redshift for the matched clusters (Figure 2). The best fit gives $\alpha=9.8 \pm 1.0$. We get similar results when matching WHL1 2 clusters in other $R_{L *}$ ranges. Figure 3 shows the comparison of $R_{L *}$ with $R_{\text {mea }}$ and $R_{\text {scal }}$. Clearly, $R_{\text {scal }}$ has a better correlation with $R_{L *}$ than with $R_{\text {mea }}$.

We define a cluster to have $R_{\text {scal }} \geq 30$ in our new identifications. To avoid the occasional projection effect, we also require $R_{\text {mea }} \geq 4$. The cluster candidates with photometric redshift of $z<0.1$ are excluded because the angular radius varies rapidly for the fixed radius of $1 \mathrm{Mpc}$ at low redshift, which induces large uncertainty on richness. Finally, we get 302 clusters in the redshift range of $0.1<z<0.35$ from the $275 \mathrm{deg}^{2}$ SDSS Stripe 82 area, which is listed in Table 1. The histograms for the redshift and scaled richness are shown in Figure 4. The identified clusters have a mean redshift of 0.18 . If our algorithm is applied to the all sky SuperCOSMOS, 2MASS and WISE data excluding the Galactic plane of $|b|>10^{\circ}$, we can find about 37,000 galaxy clusters, which will greatly enlarge the number of galaxy clusters in the region outside of the SDSS coverage. 

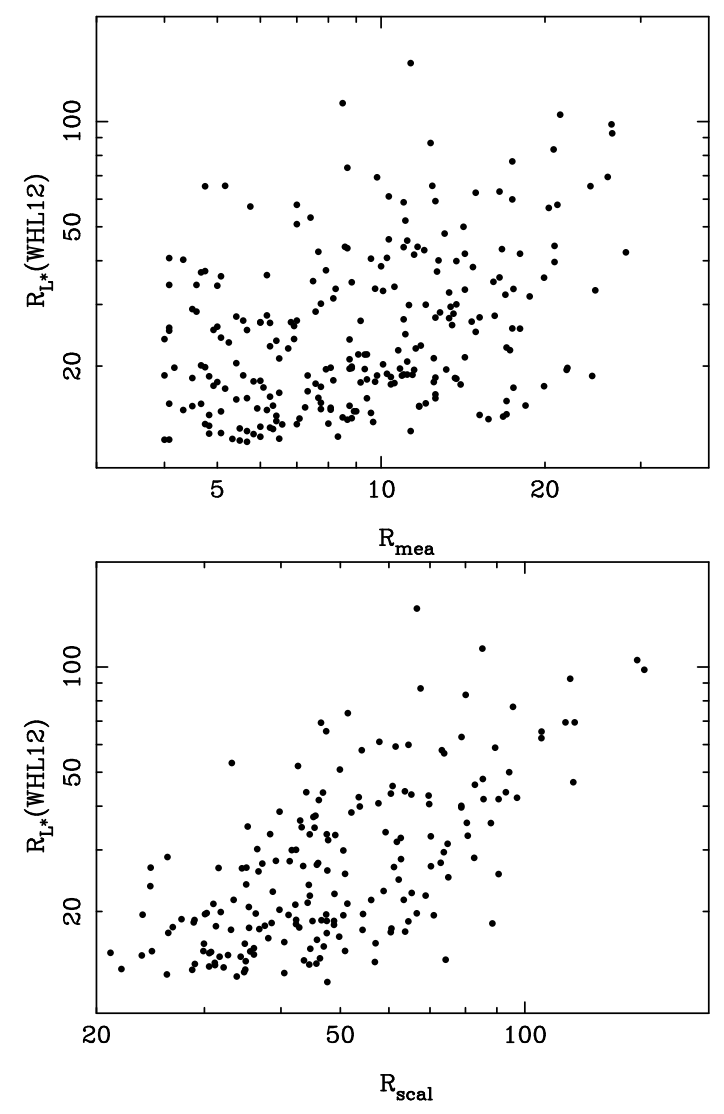

Figure 3 Comparison of measured richness $R_{\text {mea }}$ (upper panel) and scaled richness $R_{\text {scal }}$ (lower panel) with the richness in WHL12 for matched cluster candidates.

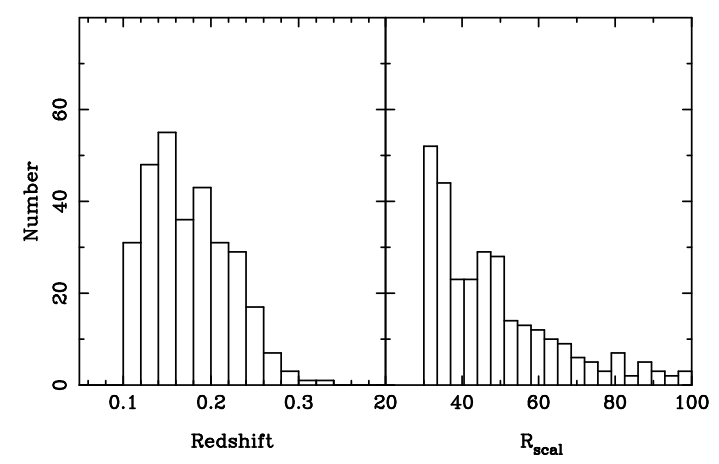

Figure 4 Distributions of redshift and scaled richness for the 302 identified clusters.

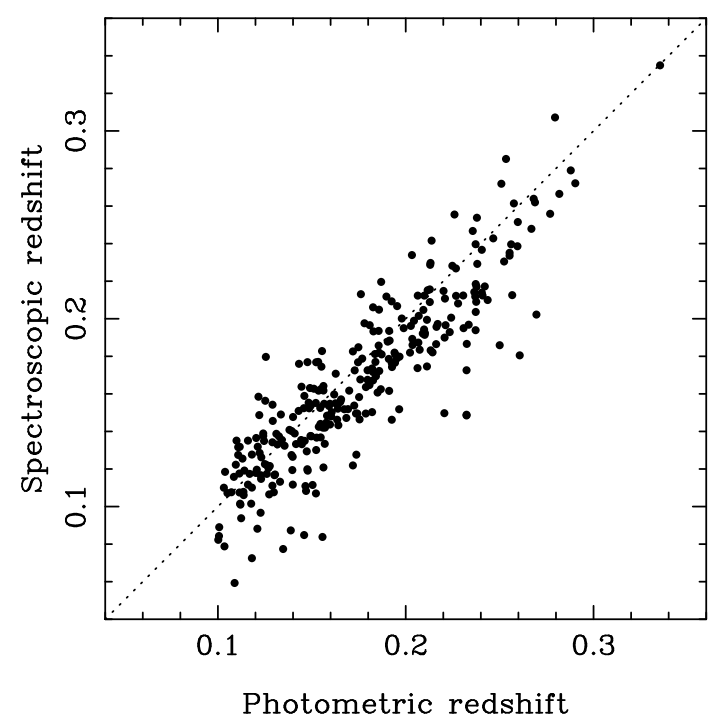

Figure 5 Comparison between cluster photometric redshift and spectroscopic redshift for 291 clusters.

To estimate the uncertainty of photometric redshift of clusters, we compare the cluster redshifts with the spectroscopic redshifts of the central galaxies in the SDSS DR7 data (Abazajian et al. 2009). 291 of 302 central galaxies have their spectroscopic redshifts measured already. As shown in Figure 5 , the cluster photometric redshift is consistent with spectroscopic redshift with a scatter of 0.022 . The central galaxies without spectroscopic redshifts are not observed by the SDSS spectroscopic survey, probably due to fiber collision. We take the value of 0.022 as the typical uncertainty of cluster photometric redshift.

\section{COMPARISON WITH PREVIOUS GALAXY CLUSTERS CATALOGS}

There are many clusters in the Stripe 82 region identified previously in the catalogs (i.e., Goto et al.2002; Koester et al. 2007; Hao et al. 2010; Szabo et al. 2011; Geach et al. 2011; Wen et al. 2009, 2012). Generally, these catalogs have a low false detection rate of $\sim 5 \%$. The completeness is as high as $>90 \%$ for clusters with mass $>1.0 \times 10^{14} M_{\odot}$ and is about $50 \%$ for cluster with mass $0.6 \times 10^{14} M_{\odot}$ (e.g., Wen et al. 2012). We regard the clusters in these catalogs as true clusters and compare them with 302 identified clusters. 247 of 302 $(82 \%)$ identified clusters are matched with the known clusters within a separation of $1 \mathrm{Mpc}$ and a redshift difference of 0.05 . The matched percentage of the identified clusters with known SDSS clusters varies with richness as expected, as shown in Figure 6 . The matched percentage increases from $82 \%$ with a richness of $R_{\text {scal }} \geq 30$ to $95 \%$ with a richness of $R_{\text {scal }} \geq 60$. Most of the unmatched clusters have a low richness which all previous methods are less sensitive to detect. In previous catalogs, the matched percentage between them is in the range of $40 \%-80 \%$, and even lower for poor clusters with a small richness (Szabo et al. 2011; Wen et al. 2012). The value of $82 \%$ is very high compared with previous matched percentage, suggesting that our identification method is very efficient for finding clusters by using the SuperCOSMOS, 2MASS and WISE data.

There are 196 clusters matched with the WHL12 clusters, of which $118(60 \%)$ have the central galaxies matched with the brightest cluster galaxies (BCG) of the WHL12, suggesting that the method presented in this paper has a high probability to find the BCGs. We calculate the projected distance 
between the central galaxies of identified clusters and BCGs of the WHL12 clusters. As shown in Figure 7, the distribution is random for the central galaxies not matched with the BCGs. For these clusters, the BCGs may not be located at the positions with the maximum overdensity of galaxy numbers found within a radius of $1 \mathrm{Mpc}$. Some of these clusters may have multiple bright member galaxies in different subclusters.

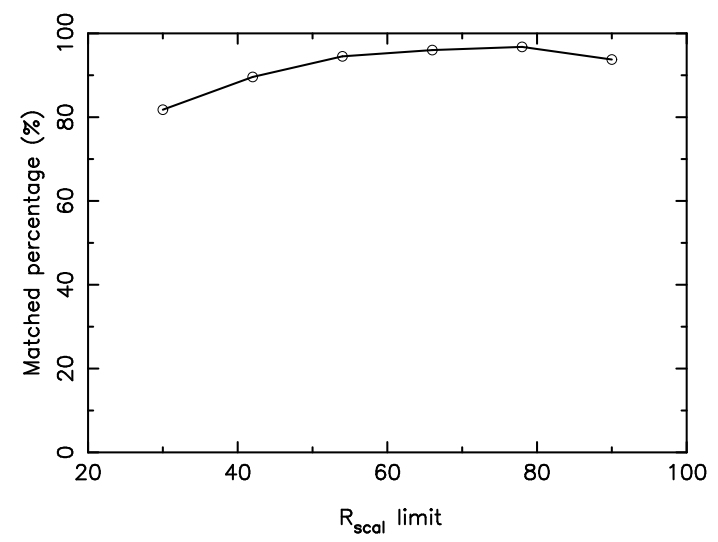

Figure 6 Matched percentage of identified clusters by previously known SDSS clusters as a function of richness limit.

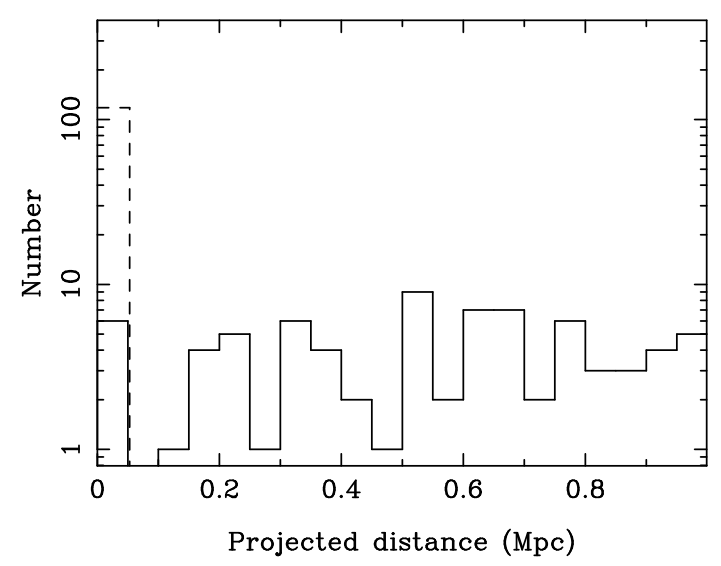

Figure 7 Distribution of projected distance between central galaxies of the identified clusters and BCGs of the WHL12 clusters. The dashed line is for the matched central galaxies with angular offset less than $3^{\prime \prime}$ from the BCGs.

Many works used red-sequence methods to identify clusters in multi-band surveys (e.g., Gladders \& Yee 2000, 2005; Koester et al. 2007). The basis of such methods is that cluster galaxies have similar colors, which are tightly related to redshift. We check if the colors by the WISE and 2MASS data have tight correlations with redshift for the central galaxies. We find that the colors, $J-W 1$ and $J-W 3$, have poor correlations with redshift (Figure 8). Cluster identification with $J-W 1$ and $J-W 3$ may have large uncertainty at redshift of $z<0.35$. Therefore the photometric redshift data provide a better opportunity to identify a whole sky galaxy cluster cata$\log$ up to redshift $z \sim 0.35$.
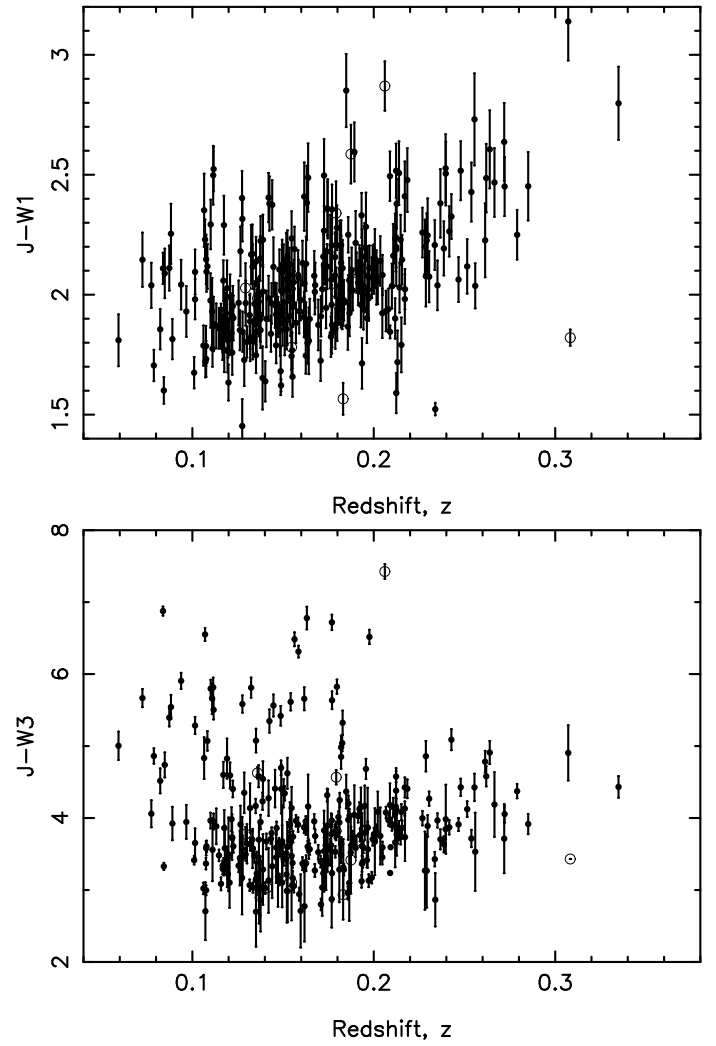

Figure 8 Colors $J-W 1$ (upper panel) and $J-W 3$ (lower panel) of central galaxies versus cluster redshift. Black dots are for spectroscopic redshifts. Open circles are for photometric redshifts with a typical uncertainty of 0.022 .

\section{SUMMARY}

In this paper, we present an efficient method to identify galaxy clusters by using the SuperCOSMOS, 2MASS and WISE data. First, we perform star-galaxy separation by color index, $W 1-J$. Then, clusters are identified around the galaxies with estimated photometric redshift. We get a measured richness and define a scaled richness, $R_{\text {scal }}$, by comparing the richness of Wen et al. (2012). Our method is applied to the data in the SDSS Stripe 82 region and identify 302 clusters of $R_{\text {scal }} \geq 30$ in the redshift range of $0.1<z<0.35$. The photometric redshift has an uncertainty of $0.022 .82 \%$ of our clusters are matched with previous SDSS cluster catalogs. Our results confirm that this approach has a good potential to detect many new galaxy clusters in the all sky data of SuperCOSMOS, 2MASS and WISE, especially in the region out of the SDSS coverage.

We thank the referee for valuable comments that helped to improve the paper. We thank Jun Han for carefully reading the manuscript, Zhongsheng Yuan and Jun Xu for useful discussion. The authors are supported by the National Natural Science Foundation (NNSF) of China (10833003 and 11103032) and the Young Researcher Grant of National Astronomical Observatories, Chinese Academy of Sciences. This publication makes use of data products from the Wide-field Infrared Survey Explorer, which is a joint project of the University of California, Los Angeles, and the Jet Propulsion Laboratory/California Institute of Technology, funded by the National Aeronautics and Space Administration. This research 
has made use of the NASA/IPAC Infrared Science Archive, which is operated by the Jet Propulsion Laboratory, California Institute of Technology, under contract with the National Aeronautics and Space Administration. Funding for the SDSS and SDSS-II has been provided by the Alfred P. Sloan Foundation, the Participating Institutions, the National Science Foundation, the U.S. Department of Energy, the National Aeronautics and Space Administration, the Japanese Monbukagakusho, the Max Planck Society, and the Higher Education Funding Council for England. The SDSS Web site is http://www.sdss.org/. The SDSS is managed by the Astrophysical Research Consortium for the Participating Institutions. The Participating Institutions are the American Museum of Natural History, Astrophysical Institute Potsdam,
University of Basel, Cambridge University, Case Western Reserve University, University of Chicago, Drexel University, Fermilab, the Institute for Advanced Study, the Japan Participation Group, Johns Hopkins University, the Joint Institute for Nuclear Astrophysics, the Kavli Institute for Particle Astrophysics and Cosmology, the Korean Scientist Group, the Chinese Academy of Sciences (LAMOST), Los Alamos National Laboratory, the Max Planck Institute for Astronomy (MPIA), the Max Planck Institute for Astrophysics (MPA), New Mexico State University, Ohio State University, University of Pittsburgh, University of Portsmouth, Princeton University, the United States Naval Observatory, and the University of Washington.

\section{REFERENCES}

Abell, G. O. 1958, ApJS, 3, 211

Abell, G. O., Corwin, H. G., Jr., \& Olowin, R. P. 1989, ApJS, 70, 1

Abazajian, K. N., Adelman-McCarthy, J. K., Agüeros, M. A., et al. 2009, ApJS, 182, 543

Allen, S. W., Evrard, A. E., \& Mantz, A. B. 2011, ARA\&A, 49, 409

Bahcall, N. A. 1988, ARA\&A, 26, 631

Bilicki, M., Jarrett, T. H., Peacock, J. A., Cluver, M. E., \& Steward, L. 2014 ApJS, 210, 9

Csabai, I., Budavári, T., Connolly, A. J., et al. 2003, AJ, 125, 580

Gal, R. R., de Carvalho, R. R., Lopes, P. A. A., et al. 2003, AJ, 125, 2064

Geach, J. E., Murphy, D. N. A., \& Bower, R. G. 2011, MNRAS, 413, 3059

Gettings, D. P., Gonzalez, A. H., Stanford, S. A., et al. 2012, ApJ, 759, L23

Gladders, M. D., \& Yee, H. K. C. 2000, AJ, 120, 2148

Gladders, M. D., \& Yee, H. K. C. 2005, ApJS, 157, 1

Goto, T., Sekiguchi, M., Nichol, R. C., et al. 2002, AJ, 123, 1807

Gunn, J. E., Hoessel, J. G., \& Oke, J. B. 1986, ApJ, 306, 30

Hambly, N. C., Irwin, M. J., \& MacGillivray, H. T. 2001, MNRAS, 326, 1295

Hao, J., McKay, T. A., Koester, B. P., et al. 2010, ApJS, 191, 254

Hong, T., Han, J. L., Wen, Z. L., Sun, L., \& Zhan, H. 2012, ApJ, 749, 81

Huchra, J. P., \& Geller, M. J. 1982, ApJ, 257, 423

Kim, R. S. J., Kepner, J. V., Postman, M., et al. 2002, AJ, 123, 20

Koester, B. P., McKay, T. A., Annis, J., et al. 2007, ApJ, 660, 221

Kovács, A., \& Szapudi, I. 2013, arXiv:1401.0156
Liang, S. M., Wen, Z. L., Han, J. L., \& Jiang, Y. Y. 2014, Sci. China Phys. Mech. Astron., in press (arXiv:1310.4885)

Liu, F., Wen, Z., Han, J., \& Meng, X. 2012, Science China Physics, Mechanics, and Astronomy, 55, 354

Oyaizu, H., Lima, M., Cunha, C. E., et al. 2008, ApJ, 674, 768

Postman, M., Lubin, L. M., Gunn, J. E., et al. 1996, AJ, 111, 615

Ramella, M., Boschin, W., Fadda, D., \& Nonino, M. 2001, A\&A, 368, 776

Reiprich, T. H., Bohringer, H. 2002, ApJ, 567, 716

Skrutskie, M. F., Cutri, R. M., Stiening, R., et al. 2006, AJ, 131, 1163

Szabo, T., Pierpaoli, E., Dong, F., Pipino, A., \& Gunn, J. 2011, ApJ, 736, 21

Wen, Z. L., Han, J. L., \& Liu, F. S. 2010, MNRAS, 407, 533

Wen, Z.-L., Han, J.-L., \& Jiang, Y.-Y. 2011, Research in Astronomy and Astrophysics, 11, 1185

Wen, Z. L., \& Han, J. L. 2011, ApJ, 734, 68

Wen, Z. L., Han, J. L., \& Liu, F. S. 2009, ApJS, 183, 197

Wen, Z. L., Han, J. L., \& Liu, F. S. 2012, ApJS, 199, 34

Wright, E. L., Eisenhardt, P. R. M., Mainzer, A. K., et al. 2010, AJ, 140, 1868

Yan, L., Donoso, E., Tsai, C.-W., et al. 2013, AJ, 145, 55

York, D. G., Adelman, J., Anderson, J. E., Jr., et al. 2000, AJ, 120, 1579

Zwicky, F., Herzog, E., \& Wild, P. 1968, Pasadena: California Institute of Technology (CIT), 1961-1968, 
TABLE 1

The 302 IDENTIFIEd Cluster CANDIDATES IN THE STRIPE 82

\begin{tabular}{|c|c|c|c|c|c|c|c|c|c|}
\hline ID & $\begin{array}{l}\text { R.A. } \\
\text { (deg) }\end{array}$ & $\begin{array}{l}\text { Decl. } \\
\text { (deg) }\end{array}$ & $z_{p}$ & $z_{s}$ & $\begin{array}{c}J \\
(\mathrm{mag})\end{array}$ & $\begin{array}{c}W 1 \\
(\mathrm{mag})\end{array}$ & $\overline{R_{\text {scal }}}$ & $\overline{R_{m e a}}$ & Note \\
\hline (1) & (2) & (3) & (4) & (5) & (6) & $(7)$ & $(8)$ & (9) & $(10)$ \\
\hline 1 & 312.7079 & -1.0438 & 0.1335 & 0.1490 & $15.53 \pm 0.10$ & $13.52 \pm 0.03$ & 30.03 & 9.08 & Geach \\
\hline 2 & 312.8900 & -0.0495 & 0.1400 & 0.1477 & $15.15 \pm 0.06$ & $13.25 \pm 0.02$ & 41.98 & 12.08 & MaxBCG,AMF,Geach,WHL12 \\
\hline 3 & 313.0400 & 0.3102 & 0.1754 & 0.1464 & $15.55 \pm 0.09$ & $13.60 \pm 0.03$ & 33.57 & 7.42 & MaxBCG,GMBCG,Geach,WHL12 \\
\hline 4 & 314.3147 & 1.2120 & 0.1788 & 0.1636 & $15.61 \pm 0.08$ & $13.81 \pm 0.03$ & 36.30 & 7.83 & New \\
\hline 5 & 315.0705 & 0.4328 & 0.1035 & 0.0788 & $15.39 \pm 0.06$ & $13.69 \pm 0.03$ & 40.71 & 15.50 & New \\
\hline 6 & 315.7259 & 0.8867 & 0.2576 & 0.2614 & $16.45 \pm 0.15$ & $14.22 \pm 0.04$ & 83.93 & 10.33 & AMF,Geach,WHL12 \\
\hline 7 & 315.7609 & 0.8942 & 0.1340 & 0.1355 & $15.07 \pm 0.07$ & $13.12 \pm 0.03$ & 62.27 & 18.75 & GMBCG,Geach,WHL12 \\
\hline 8 & 315.8023 & -1.0171 & 0.1912 & 0.1616 & $15.64 \pm 0.08$ & $13.73 \pm 0.03$ & 75.73 & 14.92 & GMBCG,AMF,Geach,WHL12 \\
\hline 9 & 315.8495 & 0.9987 & 0.1477 & 0.1769 & $16.06 \pm 0.10$ & $13.85 \pm 0.04$ & 36.20 & 9.83 & WHL12 \\
\hline 10 & 316.1830 & 0.9142 & 0.1826 & 0.1672 & $15.88 \pm 0.10$ & $13.80 \pm 0.03$ & 36.94 & 7.75 & GMBCG,AMF,Geach,WHL12 \\
\hline 11 & 317.9637 & 0.0149 & 0.2209 & 0.2107 & $16.18 \pm 0.13$ & $14.02 \pm 0.03$ & 43.97 & 7.00 & MaxBCG,GMBCG,AMF,Geach,WHL12 \\
\hline 12 & 318.5470 & -0.5487 & 0.2553 & 0.2351 & $15.95 \pm 0.10$ & $13.91 \pm 0.03$ & 31.99 & 4.00 & AMF,WHL12 \\
\hline 13 & 319.3704 & 0.1176 & 0.2553 & 0.2336 & $16.05 \pm 0.10$ & $13.84 \pm 0.03$ & 33.35 & 4.17 & MaxBCG,GMBCG,AMF,Geach,WHL12 \\
\hline 14 & 319.5089 & 0.8675 & 0.156 & 0.120 & $15.72 \pm 0.09$ & $13.73 \pm 0.03$ & 36.30 & 9.25 & Geach \\
\hline 15 & 319.9571 & 1.1862 & 0.1204 & 0.136 & $15.52 \pm 0.07$ & $13.55 \pm 0.02$ & 49.88 & 16.67 & AMF \\
\hline 16 & 320.9352 & -0.6985 & 0.2308 & 0.195 & $15.89 \pm 0.10$ & $13.69 \pm 0.03$ & 49.39 & 7.33 & MaxBCG,GMBCG,AMF,Geach,WHL12 \\
\hline 17 & 321.1893 & -0.5187 & 0.2524 & 0.230 & $15.98 \pm 0.10$ & $13.90 \pm 0.04$ & 54.87 & 7.00 & MaxBCG,GMBCG,AMF \\
\hline 18 & 321.4364 & 1.0129 & 0.1497 & 0.13 & $15.54 \pm 0.09$ & $13.64 \pm 0.03$ & 65.10 & 17.42 & GMBCG,AMF,Ge \\
\hline 19 & 322.4165 & 0.0891 & 0.2033 & 0.2339 & $14.98 \pm 9.99$ & $13.46 \pm 0.03$ & 68.29 & 12.33 & MaxBCG,GMBCG, \\
\hline 20 & 322.4345 & 0.3405 & 0.1952 & 0.1787 & $15.68 \pm 0.10$ & $13.52 \pm 0.03$ & 46.15 & 8.83 & MaxBCG,Geac \\
\hline 21 & 322.4928 & -0.3298 & 0.1547 & & $15.68 \pm 0.09$ & $13.90 \pm 0.03$ & 42.69 & 11.00 & MaxBCG, \\
\hline 22 & 322.5165 & -0.3523 & 0.2405 & 0.2367 & $16.37 \pm 0.14$ & $13.99 \pm 0.03$ & 50.52 & 7.00 & MaxBCG, \\
\hline 23 & 322.5982 & -0.0492 & 0.1569 & 0.1334 & $15.64 \pm 0$ & $13.82 \pm 0$ & 54.24 & 13.75 & MaxBCG,GMBCG,G \\
\hline 24 & 323.1762 & 1.1921 & 0.2061 & & $16.11 \pm 0.10$ & $13.24 \pm 0$ & 37.72 & 6.67 & New \\
\hline 25 & 323.5251 & -0.5225 & 0.2381 & 0.2292 & $15.78 \pm 0.11$ & $13.55 \pm 0.03$ & 53.79 & 7.58 & GMBCC \\
\hline 26 & 323.5826 & 0.5438 & 0.1967 & 0.1798 & $15.61 \pm 0.07$ & $13.73 \pm 0.03$ & 41.37 & 7.83 & GMBCC \\
\hline 27 & 323.8004 & -1.0496 & 0.3083 & $\ldots$ & $15.67 \pm 9.99$ & $13.85 \pm 0.03$ & 67.92 & 5.92 & GMBCG,Gea \\
\hline 28 & 323.9428 & 0.1158 & 0.1169 & 0.1174 & $15.81 \pm 0.12$ & $13.52 \pm 0.03$ & 120.70 & 41.42 & Abell,MaxBCG,GMBCG,A \\
\hline 29 & 324.6582 & -0.3506 & 0.152 & 0.1552 & $15.24 \pm 0.07$ & $13.58 \pm 0.05$ & 30.44 & 8.00 & New \\
\hline 30 & 326.1441 & 1.1394 & 0.2267 & 0.22 & $16.00 \pm 0$ & $13.74 \pm 0$ & 89.52 & 13.67 & Abell,Geac \\
\hline 31 & 326.3670 & -0.7800 & & & $15.36 \pm 0$ & $13.23 \pm 0$ & 73.60 & 15.1 & MaxBCG,GMBCG,G \\
\hline 32 & 326.5361 & -0.2424 & & & $16.15 \pm 0$ & $14.07 \pm 0$ & 31.67 & & \\
\hline 33 & 326.8617 & 0.7289 & & 0.072 & $15.72 \pm 0$ & $13.57 \pm 0$ & 51.21 & 17.42 & Abell,Ma \\
\hline 34 & 328.5980 & 0.0844 & 0.1603 & 0.1480 & $15.05 \pm 0$ & $12.94 \pm 0$ & 46.50 & 11.50 & MaxBCG,GMBCC \\
\hline 35 & 328.6150 & 0.6436 & 0.183 & & $14.86 \pm 0$ & $13.29 \pm 0$ & 31.11 & 6.50 & Abell,MaxBCG,GMBCG,AMF,Geach,WHL12 \\
\hline 36 & 328.7676 & 0.8724 & 0.2064 & 0.2123 & $16.14 \pm 0$ & $13.62 \pm 0$ & 58.51 & 10.33 & GMBCG,AMF,Geach,WHL12 \\
\hline 37 & 328.8372 & 1.1254 & 0.23 & 0.2118 & $15.67 \pm 0$ & $13.77 \pm 0$ & 39.91 & 5.6 & Geach,WHL12 \\
\hline 38 & 328.9167 & 0.5372 & 0.18 & 0.2048 & $15.51 \pm 0$ & $13.43 \pm 0$ & 46.75 & 9.58 & MaxBCG,AMF,Geach,WHL12 \\
\hline 39 & 329.3690 & -0.9485 & & & $16.39 \pm 0$ & $14.04 \pm$ & 32.08 & 10. & New \\
\hline 40 & 329.3874 & -0.9288 & & & $15.74 \pm 0$ & $13.70 \pm 0$ & 71.06 & 10.08 & MaxBCG,Geach,WHL12 \\
\hline 41 & 329.9931 & -0.6349 & & & $15.54 \pm 0$ & $14.09 \pm 0$ & 47.27 & 17.00 & AMF,WHL12 \\
\hline 42 & 330.1559 & -0.5459 & & & $15.44 \pm 0$ & $13.26 \pm$ & 49.43 & 14. & MaxBCG,Geach,WHL12 \\
\hline 43 & 331.2684 & -0.5626 & & & $15.73 \pm 0$ & $13.77 \pm 0$ & 69.46 & 17.2 & MaxBCG,Geach,WHL12 \\
\hline 44 & 331.6891 & 1.0279 & 0.2 & & $16.25 \pm 0.13$ & $13.74 \pm 0$ & 43.50 & 6.1 & AMF,Geach,WHL12 \\
\hline 45 & 332.3338 & 1.2364 & & & $15.64 \pm 0.09$ & $13.67 \pm 0.03$ & 37.50 & 9.25 & New \\
\hline 46 & 334.1963 & -0.9939 & 0.1965 & 0.1518 & $15.78 \pm 0.11$ & $13.64 \pm 0.03$ & 31.64 & 6.00 & WHL12 \\
\hline 47 & 335.4678 & -0.9728 & 0.3354 & 0.3349 & $16.51 \pm 0.15$ & $13.71 \pm 0.03$ & 65.23 & 4.75 & Geach \\
\hline 48 & 335.4820 & -1.0553 & 0.1 & 0.1072 & $14.89 \pm 0.06$ & $13.17 \pm 0.02$ & 64.94 & 24.42 & WHL12 \\
\hline 49 & 335.5464 & -1.0006 & & 0.1497 & $15.80 \pm 0.09$ & $13.92 \pm 0.03$ & 44.97 & $\begin{array}{c}24.42 \\
7.17\end{array}$ & New \\
\hline 50 & 335.6234 & 0.5694 & 0.1859 & 0.1722 & $15.53 \pm 0.09$ & $13.37 \pm 0.02$ & 67.16 & 13.75 & Abell,MaxBCG,Geach,WHL12 \\
\hline 51 & 335.7316 & 0.5291 & 0.1445 & 0.1638 & $16.41 \pm 0.14$ & $13.92 \pm 0.03$ & 45.54 & 12.67 & Abell,GMBCG,AMF,Geach,WHL12 \\
\hline 52 & 336.0885 & 0.3597 & 0.1570 & 0.1420 & $15.71 \pm 0.09$ & $13.73 \pm 0.03$ & 65.81 & 16.67 & MaxBCG,GMBCG,Geach,WHL12 \\
\hline 53 & 336.2299 & -0.3840 & 0.1559 & 0.1420 & $15.38 \pm 0.10$ & $12.98 \pm 0.02$ & 54.80 & 14.00 & MaxBCG,Geach,WHL12 \\
\hline 54 & 337.2881 & 0.4193 & 0.1523 & 0.1301 & $15.41 \pm 0.06$ & $13.61 \pm 0.03$ & 30.80 & 8.08 & Geach,WHL12 \\
\hline 55 & 337.5326 & -0.0037 & 0.2279 & 0.2081 & $15.72 \pm 0.08$ & $13.78 \pm 0.03$ & 86.35 & 13.08 & MaxBCG,GMBCG,AMF,Geach,WHL12 \\
\hline 56 & 337.8498 & 0.2527 & 0.2769 & 0.2559 & $15.97 \pm 0.09$ & $13.93 \pm 0.03$ & 75.82 & 8.17 & MaxBCG,GMBCG,Geach,WHL12 \\
\hline 57 & 337.9117 & -1.0345 & 0.1458 & 0.1524 & $15.94 \pm 0.11$ & $13.78 \pm 0.03$ & 31.46 & 8.67 & New \\
\hline 58 & 338.4055 & 0.6970 & 0.1742 & 0.1495 & $15.99 \pm 0.11$ & $13.87 \pm 0.03$ & 56.38 & 12.58 & AMF \\
\hline 59 & 338.5464 & 1.0411 & 0.1905 & $\ldots$ & $15.85 \pm 0.08$ & $13.76 \pm 0.03$ & 60.60 & 12.00 & MaxBCG \\
\hline 60 & 338.8853 & -1.1846 & 0.1007 & 0.0890 & $15.34 \pm 0.08$ & $13.52 \pm 0.03$ & 41.57 & 16.17 & GMBCG,Geach,WHL12 \\
\hline 61 & 339.5265 & 0.5325 & 0.2373 & 0.2036 & $15.89 \pm 0.10$ & $13.75 \pm 0.03$ & 34.71 & 4.92 & WHL12 \\
\hline 62 & 339.5725 & -1.0250 & 0.1301 & 0.1167 & $15.43 \pm 0.07$ & $13.60 \pm 0.03$ & 64.22 & 19.92 & MaxBCG,WHL12 \\
\hline 63 & 339.6037 & 0.4290 & 0.1476 & 0.1199 & $15.58 \pm 0.07$ & $13.95 \pm 0.03$ & 36.79 & 10.00 & GMBCG \\
\hline 64 & 339.6763 & -0.4495 & 0.1737 & 0.1276 & $15.82 \pm 0.09$ & $13.50 \pm 0.03$ & 44.67 & 10.00 & New \\
\hline 65 & 339.7389 & 0.9962 & 0.1241 & 0.1389 & $15.87 \pm 0.10$ & $13.86 \pm 0.03$ & 41.59 & 13.50 & New \\
\hline
\end{tabular}


TABLE 1

CONTINUED

\begin{tabular}{|c|c|c|c|c|c|c|c|c|c|}
\hline ID & $\begin{array}{l}\text { R.A. } \\
\text { (deg) }\end{array}$ & $\begin{array}{l}\text { Decl. } \\
\text { (deg) }\end{array}$ & $z_{p}$ & $z_{s}$ & $\begin{array}{c}J \\
(\mathrm{mag})\end{array}$ & $\begin{array}{c}\begin{array}{c}W 1 \\
(\mathrm{mag})\end{array}\end{array}$ & $\overline{R_{\text {scal }}}$ & $\overline{R_{m e a}}$ & Note \\
\hline (1) & (2) & (3) & $(4)$ & $(5)$ & (6) & (7) & $(8)$ & (9) & $(10)$ \\
\hline 66 & 339.7720 & 0.6534 & 0.2375 & 0.2090 & 15.60 & $13.76 \pm 0.03$ & 54.20 & 7.67 & MaxBCG,Geach,WHL12 \\
\hline 67 & 339.9143 & 1.1757 & 0.1641 & 0.1433 & $15.70 \pm 0.07$ & $13.86 \pm 0.03$ & 45.42 & 10.92 & Geach,WHL12 \\
\hline 68 & 340.6530 & 0.8837 & 0.1222 & 0.1286 & $15.65 \pm 0.10$ & $13.92 \pm 0.04$ & 50.59 & 16.67 & New \\
\hline 69 & 340.8411 & 0.3386 & 0.1089 & 0.0593 & $15.78 \pm 0.10$ & $13.97 \pm 0.04$ & 46.78 & 17.08 & WHL12 \\
\hline 70 & 341.1638 & 0.5700 & 0.1137 & 0.1061 & $15.02 \pm 0.08$ & $13.23 \pm 0.02$ & 44.77 & 15.75 & New \\
\hline 71 & 341.7559 & 0.9144 & 0.1793 & $\ldots$ & $15.81 \pm 0.10$ & $13.80 \pm 0.03$ & 34.92 & 7.50 & MaxBCG,Geach \\
\hline 72 & 342.0809 & -0.6117 & 0.2099 & 0.2123 & $15.90 \pm 0.08$ & $13.70 \pm 0.03$ & 46.02 & 7.92 & GMBCG,AMF,WHL12 \\
\hline 73 & 342.1753 & 0.9131 & 0.1359 & $\ldots$ & $15.13 \pm 0.08$ & $13.15 \pm 0.03$ & 38.73 & 11.50 & MaxBCG,AMF \\
\hline 74 & 342.5280 & 0.8538 & 0.1927 & 0.1743 & $15.68 \pm 0.09$ & $13.56 \pm 0.03$ & 47.87 & 9.33 & MaxBCG,GMBCG,AMF,Geach,WHL12 \\
\hline 75 & 342.7946 & -0.7928 & 0.2365 & 0.2142 & $15.92 \pm 0.10$ & $13.89 \pm 0.03$ & 38.59 & 5.50 & Geach,WHL12 \\
\hline 76 & 342.9160 & 1.1677 & 0.1388 & 0.0873 & $15.65 \pm 0.09$ & $13.54 \pm 0.03$ & 30.98 & 9.00 & New \\
\hline 77 & 343.9557 & 0.5725 & 0.1910 & 0.1786 & $15.85 \pm 0.09$ & $13.77 \pm 0.03$ & 35.07 & 6.92 & New \\
\hline 78 & 344.0618 & -0.5811 & 0.1032 & 0.1100 & $15.93 \pm 0.11$ & $13.95 \pm 0.03$ & 81.27 & 31.00 & Abell,MaxBCG,GMBCG,AMF,Geach,WHL12 \\
\hline 79 & 344.1515 & -0.4658 & 0.1469 & 0.1083 & $15.55 \pm 0.09$ & $13.43 \pm 0.03$ & 74.41 & 20.33 & Abell,MaxBCG,GMBCG,AMF,Geach,WHL12 \\
\hline 80 & 344.3402 & -1.1362 & 0.1792 & $\ldots$ & $15.82 \pm 0.13$ & $13.48 \pm 0.03$ & 38.00 & 8.17 & MaxBCG,Geach,WHL12 \\
\hline 81 & 344.5970 & 0.2678 & 0.1291 & 0.1542 & $15.89 \pm 0.09$ & $13.85 \pm 0.03$ & 57.84 & 18.08 & MaxBCG,WHL12 \\
\hline 82 & 344.6125 & -0.1018 & 0.1872 & 0.1810 & $15.72 \pm 0.09$ & $13.74 \pm 0.03$ & 54.62 & 11.08 & MaxBCG,Geach \\
\hline 83 & 344.6595 & 0.5849 & 0.1252 & 0.1563 & $15.67 \pm 0.09$ & $13.48 \pm 0.03$ & 32.60 & 10.50 & New \\
\hline 84 & 344.7387 & 1.2057 & 0.1085 & 0.1158 & $15.38 \pm 0.09$ & $13.51 \pm 0.03$ & 47.80 & 17.50 & New \\
\hline 85 & 345.0132 & 1.1510 & 0.1304 & 0.1171 & $15.73 \pm 0.08$ & $13.67 \pm 0.03$ & 40.63 & 12.58 & New \\
\hline 86 & 345.0288 & 0.2221 & 0.1874 & $\ldots$ & $15.62 \pm 0.12$ & $13.03 \pm 0.02$ & 62.11 & 12.58 & GMBCG,AMF,Geach,WHL12 \\
\hline 87 & 345.0460 & 0.3640 & 0.2248 & 0.2282 & $15.70 \pm 0.11$ & $13.50 \pm 0.03$ & 35.00 & 5.42 & WHL12 \\
\hline 88 & 345.5182 & 0.2212 & 0.1293 & $\ldots$ & $15.79 \pm 0.10$ & $13.76 \pm 0.03$ & 33.39 & 10.42 & MaxBCG,GMBCG,Geach,WHL12 \\
\hline 89 & 345.9451 & 0.7804 & 0.1566 & 0.1547 & $15.84 \pm 0.11$ & $13.60 \pm 0.03$ & 39.66 & 10.08 & MaxBCG \\
\hline 90 & 348.1483 & 0.1520 & 0.1260 & 0.1174 & $15.03 \pm 0.08$ & $13.09 \pm 0.03$ & 44.52 & 14.25 & MaxBCG,Geach,WHL12 \\
\hline 91 & 350.2303 & 0.5441 & 0.2067 & 0.1873 & $15.73 \pm 0.09$ & $13.71 \pm 0.03$ & 62.91 & 11.08 & MaxBCG,Geach,WHL12 \\
\hline 92 & 350.6064 & 1.0693 & 0.1396 & 0.1194 & $15.09 \pm 0.08$ & $13.32 \pm 0.04$ & 30.30 & 8.75 & CE,MaxBCG,AMF,Geach,WHL12 \\
\hline 93 & 350.7556 & 0.8997 & 0.1269 & 0.1205 & $15.21 \pm 0.07$ & $13.39 \pm 0.03$ & 41.43 & 13.17 & MaxBCG,AMF,WHL12 \\
\hline 94 & 351.0899 & 0.3193 & 0.1735 & 0.1495 & $15.11 \pm 0.08$ & $12.99 \pm 0.03$ & 88.83 & 19.92 & Abell,CE,MaxBCG,GMBCG,AMF,Geach,WHL12 \\
\hline 95 & 351.1997 & 0.9442 & 0.1038 & 0.1185 & $15.22 \pm 0.08$ & $13.30 \pm 0.03$ & 30.94 & 11.75 & CE,WHL12 \\
\hline 96 & 354.4262 & 0.3038 & 0.1207 & 0.1196 & $15.17 \pm 0.07$ & $13.36 \pm 0.03$ & 77.26 & 25.75 & Abell \\
\hline 97 & 355.0894 & 0.2696 & 0.1447 & 0.1332 & $15.38 \pm 0.09$ & $13.26 \pm 0.02$ & 32.41 & 9.00 & $\mathrm{CE}$ \\
\hline 98 & 355.2489 & 0.0817 & 0.1748 & 0.1848 & $15.66 \pm 0.15$ & $12.81 \pm 0.03$ & 119.64 & 26.58 & Abell,CE,MaxBCG,GMBCG,AMF,Geach,WHL12 \\
\hline 99 & 355.9156 & 0.4243 & 0.2500 & 0.1859 & $15.66 \pm 0.07$ & $13.41 \pm 0.02$ & 50.10 & 6.50 & New \\
\hline 100 & 356.0726 & -0.8548 & 0.2607 & 0.1805 & $15.87 \pm 0.08$ & $13.91 \pm 0.03$ & 33.87 & 4.08 & $\mathrm{Ne}$ \\
\hline 101 & 356.5196 & -0.1857 & 0.2818 & 0.266 & $5 \pm 0$. & $13.98 \pm 0$ & 48.76 & 5.08 & CE,MaxBCG,GMBCC \\
\hline 102 & 356.5763 & 0.9610 & 0.1355 & 0.1324 & $16.06 \pm 0.12$ & $13.89 \pm 0.03$ & 32.48 & 9.6 & Geach, $\mathrm{V}$ \\
\hline 103 & 356.8651 & -0.1538 & 0.268 & 0.26 & $6.63 \pm 0$. & $14.02 \pm 0$ & 35.66 & $4 .($ & CE,GMBCG,AM \\
\hline 104 & 358.7088 & -0.1407 & 0.18 & 0.19 & $2 \pm 0$ & $32 \pm 0$ & 36.48 & 7. & WH \\
\hline 105 & 359.8242 & 0.3167 & 0.1181 & 0.1101 & $16.01 \pm 0.10$ & $13.72 \pm 0.03$ & 31.60 & 10.75 & $\mathrm{~N}$ \\
\hline 106 & 0.1008 & -1.2457 & 0.15 & 0.16 & $6 \pm 0.07$ & $13.21 \pm 0$ & 32.69 & 8.3 & New \\
\hline 107 & 0.2157 & -1.1 & 0.21 & 0.19 & \pm 0 & $8 \pm$ & 30.55 & $5 .($ & New \\
\hline 108 & 0.3598 & -0.0288 & 0.2669 & 0.2479 & $1 \pm 0.12$ & $13.69 \pm 0$ & 49.82 & 5.75 & CE,MaxBCG,GMBCG,AMF,Geach,WHL12 \\
\hline 109 & 1.1300 & -1.1274 & 0.1768 & 0.1788 & $2 \pm 0.08$ & $13.96 \pm 0$ & 35.78 & 7.8 & \\
\hline 110 & 1.8501 & -0.7548 & 0.23 & 0.186 & $94 \pm 0$ & $02 \pm 0$ & 51.15 & 7.50 & CE,G \\
\hline 111 & 1.9482 & -0.7442 & 0.1522 & 0.1070 & $15.67 \pm 0.08$ & $13.44 \pm 0.03$ & 34.92 & 9.17 & WHL12 \\
\hline 112 & 2.1160 & -0.0048 & 0.1216 & 0.1585 & $15.45 \pm 0.07$ & $13.38 \pm 0.03$ & 33.75 & 11.17 & New \\
\hline 113 & 2.4194 & 0.4251 & 0.1904 & 0.1878 & $15.74 \pm 0.09$ & $13.64 \pm 0.03$ & 35.32 & 7.00 & CE,Geach,WHL12 \\
\hline 114 & 3.0125 & -1.0070 & 0.1459 & 0.0848 & $15.71 \pm 0.10$ & $13.62 \pm 0.03$ & 33.60 & 9.25 & New \\
\hline 115 & 3.1982 & 0.7877 & 0.1581 & 0.1484 & $15.60 \pm 0.10$ & $13.75 \pm 0.04$ & 40.10 & 10.08 & MaxBCG,WHL12 \\
\hline 116 & 3.2122 & 0.2891 & 0.1430 & 0.1509 & $16.03 \pm 0.10$ & $14.17 \pm 0.04$ & 47.98 & 13.50 & CE,MaxBCG,GMBCG,AMF,WHL12 \\
\hline 117 & 3.3711 & 0.6735 & 0.1006 & 0.0843 & $14.98 \pm 0.05$ & $13.38 \pm 0.02$ & 34.88 & 13.58 & $\mathrm{CE}$ \\
\hline 118 & 4.1540 & -0.5023 & 0.1393 & 0.1402 & $15.40 \pm 0.08$ & $13.76 \pm 0.03$ & 44.66 & 12.92 & $\mathrm{CE}$ \\
\hline 119 & 4.2262 & -1.0625 & 0.2098 & 0.1943 & $15.43 \pm 0.08$ & $13.49 \pm 0.03$ & 30.02 & 5.17 & CE,MaxBCG,GMBCG,AMF,Geach,WHL12 \\
\hline 120 & 4.2529 & -1.2286 & 0.1536 & 0.1617 & $15.54 \pm 0.08$ & $13.61 \pm 0.03$ & 47.79 & 12.42 & GMBCG,AMF,WHL12 \\
\hline 121 & 4.4067 & -0.8784 & 0.2306 & 0.2124 & $16.42 \pm 0.15$ & $14.23 \pm 0.03$ & 95.39 & 14.17 & CE,MaxBCG,GMBCG,AMF,Geach,WHL12 \\
\hline 122 & 4.6355 & -0.7675 & 0.2102 & 0.1924 & $15.85 \pm 0.09$ & $13.75 \pm 0.03$ & 48.02 & 8.25 & CE,MaxBCG,GMBCG,Geach,WHL12 \\
\hline 123 & 5.0673 & 0.0794 & 0.2409 & 0.2124 & $15.21 \pm 0.06$ & $13.62 \pm 0.06$ & 154.31 & 21.33 & MaxBCG,GMBCG,AMF,Geach,WHL12 \\
\hline 124 & 5.0890 & -0.2531 & 0.2436 & 0.2100 & $15.75 \pm 0.09$ & $13.71 \pm 0.03$ & 70.66 & 9.58 & MaxBCG,AMF,Geach,WHL12 \\
\hline 125 & 5.1726 & -1.1764 & 0.1989 & 0.1950 & $15.83 \pm 0.10$ & $13.80 \pm 0.03$ & 37.14 & 6.92 & MaxBCG,Geach,WHL12 \\
\hline 126 & 5.2040 & 0.1822 & 0.2405 & 0.2139 & $16.31 \pm 0.13$ & $13.80 \pm 0.03$ & 107.68 & 14.92 & MaxBCG,GMBCG,WHL12 \\
\hline 127 & 5.3191 & -0.8368 & 0.1115 & 0.1075 & $15.40 \pm 0.08$ & $13.61 \pm 0.03$ & 117.18 & 41.92 & Abell,CE,MaxBCG,GMBCG,Geach,WHL12 \\
\hline 128 & 5.3476 & -0.8259 & 0.1797 & 0.1675 & $15.40 \pm 0.09$ & $13.39 \pm 0.03$ & 121.72 & 26.08 & CE,MaxBCG,GMBCG,AMF,Geach \\
\hline 129 & 5.6779 & -0.6892 & 0.1867 & 0.1625 & $15.74 \pm 0.07$ & $13.99 \pm 0.03$ & 37.24 & 7.58 & MaxBCG,Geach,WHL12 \\
\hline 130 & 5.7616 & -0.1367 & 0.1726 & 0.1538 & $15.90 \pm 0.11$ & $13.91 \pm 0.03$ & 107.41 & 24.25 & Abell,CE,MaxBCG,AMF,Geach,WHL12 \\
\hline
\end{tabular}


TABLE 1

CONTINUED

\begin{tabular}{|c|c|c|c|c|c|c|c|c|c|}
\hline (1) & $\begin{array}{l}\text { R.A. } \\
\text { (deg) } \\
\text { (2) }\end{array}$ & $\begin{array}{c}\text { Decl. } \\
\text { (deg) } \\
\text { (3) }\end{array}$ & (4) & (5) & $\begin{array}{c}J \\
\text { (mag) } \\
(6)\end{array}$ & $\begin{array}{c}W 1 \\
(\mathrm{mag}) \\
(7)\end{array}$ & $R_{\text {scal }}$ & $R_{\text {mea }}$ & Note \\
\hline 131 & 6.1235 & -1.0774 & 0.1311 & 0.1387 & $15.99 \pm 0.12$ & $14.34 \pm 0.05$ & 30.58 & 9.42 & Geach \\
\hline 132 & 6.3241 & -0.7247 & 0.1511 & 0.1627 & $15.78 \pm 0.11$ & $13.65 \pm 0.03$ & 30.51 & 8.08 & CE,MaxBCG,GMBCG,Geach,WHL12 \\
\hline 133 & 6.6855 & 1.2357 & 0.1673 & 0.1518 & $15.58 \pm 0.10$ & $13.52 \pm 0.03$ & 49.33 & 11.58 & Geach,WHL12 \\
\hline 134 & 7.1900 & -0.0595 & 0.1761 & 0.2131 & $16.01 \pm 0.12$ & $14.29 \pm 0.05$ & 52.29 & 11.50 & CE,MaxBCG,GMBCG,AMF,Geach,WHL12 \\
\hline 135 & 7.2203 & -0.2431 & 0.1504 & 0.1115 & $15.98 \pm 0.12$ & $13.48 \pm 0.03$ & 57.92 & 15.42 & $\mathrm{CE}$ \\
\hline 136 & 7.2757 & 0.8811 & 0.1131 & 0.1255 & $15.36 \pm 0.09$ & $13.39 \pm 0.03$ & 30.65 & 10.83 & New \\
\hline 137 & 7.4465 & 0.4922 & 0.2091 & 0.1921 & $15.91 \pm 0.09$ & $13.80 \pm 0.03$ & 48.12 & 8.33 & WHL12 \\
\hline 138 & 8.0469 & -0.6669 & 0.2201 & 0.2148 & $16.08 \pm 0.10$ & $13.85 \pm 0.03$ & 79.63 & 12.75 & CE,MaxBCG,AMF,Geach, \\
\hline 139 & 8.5832 & 0.8158 & 0.2035 & 0.1893 & $16.23 \pm 0.12$ & $13.64 \pm 0.03$ & 96.65 & 17.42 & CE,MaxBCG,GMBCG,AMF,Geach,WHL12 \\
\hline 140 & 8.7574 & -1.2049 & 0.1897 & 0.2118 & $15.94 \pm 0.10$ & $13.71 \pm 0.03$ & 59.40 & 11.83 & CE,Geach,WHL12 \\
\hline 141 & 8.7992 & 0.7300 & 0.2688 & 0.2620 & $16.47 \pm 0.14$ & $13.98 \pm 0.03$ & 49.00 & 5.58 & CE,GMBCG,Gea \\
\hline 142 & 8.8420 & 1.0672 & 0.2101 & 0.1914 & $15.83 \pm 0.09$ & $13.78 \pm 0.03$ & 31.98 & 5.50 & Geach,WH \\
\hline 143 & 9.2985 & 0.0967 & 0.2260 & 0.2555 & $16.44 \pm 0.19$ & $13.71 \pm 0.03$ & 83.61 & 12.83 & Geach,W \\
\hline 144 & 9.6975 & -1.1918 & 0.1954 & 0.2067 & $15.93 \pm 0.08$ & $14.00 \pm 0.03$ & 49.31 & 9.42 & CE,WHL \\
\hline 145 & 9.7217 & -0.2841 & 0.1858 & 0.1820 & $15.86 \pm 0.10$ & $13.65 \pm 0.03$ & 47.97 & 9.83 & CE \\
\hline 146 & 9.9280 & 0.6277 & 0.1926 & 0.1462 & $15.76 \pm 0.08$ & $13.84 \pm 0.03$ & 30.35 & 5.92 & New \\
\hline 147 & 9.9306 & -0.9176 & 0.1072 & 0.1076 & $15.95 \pm 0.10$ & $13.80 \pm 0.03$ & 43.49 & 16.08 & CE,WHL12 \\
\hline 148 & 10.8143 & 0.2296 & 0.2117 & 0.2152 & $15.97 \pm 0.11$ & $14.18 \pm 0.03$ & 41.65 & 7.08 & MaxBCG,C \\
\hline 149 & 10.8270 & -0.3129 & 0.1545 & 0.1525 & $15.15 \pm 0.08$ & $13.02 \pm 0.02$ & 37.77 & 9.75 & CE,Geach \\
\hline 150 & 10.8951 & 0.1760 & 0.1693 & 0.1519 & $15.43 \pm 0.08$ & $13.57 \pm 0.03$ & 86.85 & 20.08 & Geach \\
\hline 151 & 10.8962 & 1.0196 & 0.2164 & 0.1957 & $15.91 \pm 0.14$ & $13.63 \pm 0.03$ & 56.77 & 9.33 & BCG,AMF, \\
\hline 152 & 11.0052 & 0.1145 & 0.1869 & $0.21^{\prime}-x-1$ & $16.19 \pm 0.14$ & $13.78 \pm 0$ & 81.20 & 16.50 & CE,MaxBCG,GMBCC \\
\hline 153 & 11.0195 & 1.0314 & 0.1160 & 0.1117 & $15.43 \pm 0.09$ & $12.91 \pm 0.02$ & 43.90 & 15. & Geach, \\
\hline 154 & 11.0375 & 1.1917 & 0.1478 & 0.1191 & $15.76 \pm 0.11$ & $13.73 \pm 0$ & 46.37 & 12. & WHI \\
\hline 155 & 11.1551 & -0.9223 & 0.2242 & 0.2006 & $15.95 \pm 0.12$ & $13.80 \pm 0$ & 39.69 & 6.17 & CE,MaxBCG,GMBCG,Ge \\
\hline 156 & 11.3335 & 0.7793 & 0.1466 & 0.1109 & $16.13 \pm 0.13$ & $14.20 \pm 0$ & 34.97 & 9.58 & New \\
\hline 157 & 11.3770 & -0.7964 & 0.168 & 0.14 & $15.10 \pm 0.08$ & $13.19 \pm$ & 47.26 & 11.00 & Abell,CE,MaxI \\
\hline 158 & 11.5601 & 0.0004 & 0.13 & 0.11 & $15.88 \pm 0$ & $0 \pm$ & 97.81 & 28. & CE,MaxBC \\
\hline 159 & 11.5934 & -0.1551 & 0.2373 & 0.21 & $16.15 \pm 0.13$ & $7 \pm$ & 61.17 & & CE,MaxB \\
\hline 160 & 11.8588 & -0.9482 & 0.152 & 0.17 & $15.86 \pm 0$ & $2 \pm$ & 79.39 & 20. & \\
\hline 161 & 12.9848 & -1.0960 & 0.12 & 0.1 & $15.20 \pm 0$ & $4 \pm$ & 35.70 & & \\
\hline 162 & 13.4089 & 0.9207 & 0.2 & & $.17 \pm 0$ & $2 \pm$ & 61.19 & & \\
\hline 163 & 13.4427 & -0.7802 & 0.12 & & $15.39 \pm 0$ & $7 \pm$ & 64.17 & 20. & \\
\hline 164 & 13.8141 & -0.3482 & 0.1 & & $4 \pm 0$ & $5 \pm$ & 51.77 & 12. & $\mathrm{CE}, \mathrm{MaxBCC}$ \\
\hline 165 & 14.3250 & 0.0257 & 0.19 & 0.19 & $5 \pm 0$. & $9 \pm($ & 54.97 & 10. & \\
\hline 166 & 15.2043 & -0.4221 & 0.2 & 0.2022 & $6 \pm 0$ & $1 \pm$ & 60.31 & & $\mathrm{Ne}$ \\
\hline 167 & 15.2281 & -1.1552 & 0.1412 & & $15.00 \pm 9$ & \pm & 47.60 & 13. & New \\
\hline 168 & 15.3084 & 0.5743 & 0.21 & 0.19 & $33 \pm 0.09$ & $2 \pm$ & 70.42 & 12.00 & CE,MaxBCG,GMBCG,AN \\
\hline 169 & 5.3301 & -0.0553 & & & $7 \pm 0.09$ & $5 \pm$ & 56. & & CE \\
\hline 170 & 5.3609 & -0.0636 & 0.1 & & $0 \pm 0.08$ & \pm & 48.06 & 16. & Abel \\
\hline 171 & 5.3715 & -0.2619 & 0.22 & 0.19 & $1 \pm 0.09$ & $5 \pm 0$ & 36.26 & & \\
\hline 172 & 5.3876 & 0.5376 & 0.11 & & $8 \pm 0.09$ & \pm & 35.13 & 12. & \\
\hline 173 & 5.4146 & -0.2248 & 0.12 & & $6 \pm 0.07$ & \pm 0 & 50.59 & & Abell,CE \\
\hline 174 & 5.4679 & 0.6898 & 0.1292 & 0.14 & $4 \pm 0.09$ & $8 \pm 0$ & 30.16 & & \\
\hline 175 & 15.6797 & 1.1362 & 0.1546 & 0.14 & $15.41 \pm 0.10$ & $3 \pm 0$ & 65.92 & 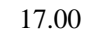 & CE,MaxBC \\
\hline 176 & 15.7192 & 0.2479 & 0.2094 & 0.2047 & $15.79 \pm 0.10$ & $7 \pm 0$ & 61.22 & 10.58 & \\
\hline 177 & 16.0185 & -0.4345 & & & $16.04 \pm 0.10$ & $9 \pm 0$ & 46.72 & 4.67 & $\mathrm{CE}, \mathrm{I}$ \\
\hline 178 & 16.2306 & 0.0602 & & & $16.24 \pm 0.12$ & $9 \pm 0$ & 86.44 & & CE,MaxBCG,GMB \\
\hline 179 & 16.6342 & 0.6310 & 0.1578 & & $16.13 \pm 0.12$ & $14.01 \pm 0.03$ & 38.73 & 9.75 & WHL12 \\
\hline 180 & 16.8165 & 0.6614 & & & $15.55 \pm 0.08$ & $13.64 \pm 0.03$ & 37.97 & 10.17 & $\mathrm{CE}$ \\
\hline 181 & 16.8618 & 0.1470 & 0.2597 & & $16.11 \pm 0.11$ & $13.99 \pm 0.03$ & 46.03 & 5.58 & CE,MaxBCG,AMF,Geach,WHL12 \\
\hline 182 & 16.9189 & 1.0481 & 0.1483 & & $15.52 \pm 0.10$ & $13.49 \pm 0.03$ & 31.44 & & WHL12 \\
\hline 183 & 17.3901 & -0.8986 & 0.2063 & 0.1737 & $15.45 \pm 0.08$ & $13.51 \pm 0.03$ & 59.87 & 10.58 & CE,Geach,WHL12 \\
\hline 184 & 17.4051 & -0.9297 & 0.1210 & & $16.16 \pm 0.12$ & $13.91 \pm 0.04$ & 59.65 & 19.83 & New \\
\hline 185 & 17.6656 & 1.0666 & 0.1926 & 0.1764 & $15.44 \pm 0.08$ & $13.61 \pm 0.03$ & 44.86 & 8.75 & CE,MaxBCG,AMF,WHL12 \\
\hline 186 & 17.9198 & -0.7625 & 0.1116 & 0.1318 & $15.79 \pm 0.11$ & $13.90 \pm 0.03$ & 43.14 & 15.42 & Geach,WHL12 \\
\hline 187 & 17.9535 & -0.0181 & 0.2379 & 0.2538 & $15.89 \pm 0.12$ & $13.46 \pm 0.03$ & 97.41 & 13.75 & CE,MaxBCG,GMBCG,Geach,WHL12 \\
\hline 188 & 18.1542 & -0.6651 & 0.2357 & 0.2467 & $15.85 \pm 0.09$ & $13.79 \pm 0.03$ & 52.88 & 7.58 & MaxBCG,Geach \\
\hline 189 & 18.2290 & 1.0638 & 0.1416 & 0.1333 & $15.62 \pm 0.07$ & $13.81 \pm 0.03$ & 34.01 & 9.67 & New \\
\hline 190 & 18.2797 & -0.0010 & 0.2128 & 0.2156 & $15.99 \pm 0.10$ & $13.84 \pm 0.03$ & 30.13 & 5.08 & Geach,WHL12 \\
\hline 191 & 18.5621 & -0.9125 & 0.2074 & 0.1835 & $15.56 \pm 0.08$ & $13.58 \pm 0.03$ & 55.63 & 9.75 & CE,MaxBCG,GMBCG,Geach,WHL12 \\
\hline 192 & 18.6568 & -0.8458 & 0.2023 & 0.1820 & $15.94 \pm 0.09$ & $13.98 \pm 0.03$ & 61.46 & 11.17 & GMBCG,AMF,WHL12 \\
\hline 193 & 18.8958 & -0.4914 & 0.1554 & 0.1828 & $15.98 \pm 0.11$ & $13.87 \pm 0.04$ & 35.77 & 9.17 & Geach,WHL12 \\
\hline 194 & 19.1019 & -0.1334 & 0.1943 & 0.1766 & $15.45 \pm 0.09$ & $13.39 \pm 0.03$ & 45.84 & 8.83 & CE,MaxBCG,Geach,WHL12 \\
\hline 195 & 19.4654 & -1.2269 & 0.2377 & 0.2171 & $15.97 \pm 0.10$ & $13.99 \pm 0.03$ & 37.13 & 5.25 & Geach,WHL12 \\
\hline
\end{tabular}


TABLE 1

CONTINUED

\begin{tabular}{|c|c|c|c|c|c|c|c|c|c|}
\hline ID & $\begin{array}{l}\text { R.A. } \\
\text { (deg) }\end{array}$ & $\begin{array}{l}\text { Decl. } \\
\text { (deg) }\end{array}$ & $z_{p}$ & $z_{s}$ & $\begin{array}{c}J \\
(\mathrm{mag})\end{array}$ & $\begin{array}{c}\begin{array}{c}W 1 \\
(\mathrm{mag})\end{array}\end{array}$ & $\overline{R_{\text {scal }}}$ & $\overline{R_{m e a}}$ & Note \\
\hline (1) & (2) & (3) & (4) & (5) & (6) & (7) & (8) & (9) & (10) \\
\hline 196 & 19.7274 & -1.0984 & 0.1276 & 0.1216 & $15.02 \pm 0.07$ & $13.18 \pm 0.03$ & 35.83 & 11.33 & New \\
\hline 197 & 19.7734 & -1.2336 & 0.1260 & 0.1220 & $15.21 \pm 0.08$ & $13.45 \pm 0.05$ & 55.45 & 17.75 & New \\
\hline 198 & 19.8598 & -0.7436 & 0.2421 & 0.2172 & $15.68 \pm 0.08$ & $13.66 \pm 0.03$ & 39.56 & 5.42 & CE,MaxBCG,Geach,WHL12 \\
\hline 199 & 19.8798 & -1.1572 & 0.2036 & 0.1860 & $16.07 \pm 0.11$ & $14.10 \pm 0.03$ & 74.52 & 13.42 & CE,Geach,WHL12 \\
\hline 200 & 20.1750 & -0.6050 & 0.1124 & 0.0938 & $15.88 \pm 0.10$ & $13.84 \pm 0.03$ & 37.53 & 13.33 & WHL12 \\
\hline 201 & 20.4632 & -0.1918 & 0.1979 & 0.2001 & $15.69 \pm 0.10$ & $13.58 \pm 0.03$ & 31.96 & 6.00 & CE,AMF,Geach,WHL12 \\
\hline 202 & 20.4820 & 0.0662 & 0.1347 & 0.0774 & $15.24 \pm 0.09$ & $13.20 \pm 0.03$ & 32.29 & 9.67 & New \\
\hline 203 & 20.5108 & 0.3345 & 0.1551 & 0.1745 & $15.53 \pm 0.11$ & $13.17 \pm 0.03$ & 80.74 & 20.75 & Abell,CE,MaxBCG,GMBCG,AMF,Geach,WHL12 \\
\hline 204 & 20.6525 & -0.8140 & 0.1797 & 0.1726 & $15.65 \pm 0.10$ & $13.38 \pm 0.03$ & 63.38 & 13.58 & CE,MaxBCG,GMBCG,AMF,Geach,WHL12 \\
\hline 205 & 20.8281 & 1.1489 & 0.1298 & 0.1076 & $15.80 \pm 0.07$ & $14.07 \pm 0.03$ & 35.12 & 10.92 & New \\
\hline 206 & 21.0764 & -1.2405 & 0.1728 & 0.1725 & $15.56 \pm 0.08$ & $13.43 \pm 0.02$ & 51.02 & 11.50 & GMBCG,Geach,WHL12 \\
\hline 207 & 21.2476 & -0.8441 & 0.1627 & 0.1707 & $15.68 \pm 0.08$ & $13.95 \pm 0.03$ & 32.61 & 7.92 & New \\
\hline 208 & 21.5269 & 1.2269 & 0.1925 & 0.2093 & $15.48 \pm 9.99$ & $14.56 \pm 0.05$ & 35.45 & 6.92 & CE,AMF,Geach,WHL12 \\
\hline 209 & 21.7496 & -0.7839 & 0.1807 & 0.1647 & $15.66 \pm 0.08$ & $13.76 \pm 0.03$ & 31.37 & 6.67 & New \\
\hline 210 & 23.2450 & 0.9407 & 0.1250 & 0.1226 & $15.38 \pm 0.09$ & $13.48 \pm 0.03$ & 42.62 & 13.75 & CE,MaxBCG,Geach,WHL12 \\
\hline 211 & 23.7048 & -0.6121 & 0.1557 & 0.0838 & $15.10 \pm 0.06$ & $12.99 \pm 0.03$ & 38.10 & 9.75 & CE,GMBCG,AMF,Geach,WHL12 \\
\hline 212 & 23.7313 & 0.3878 & 0.1592 & 0.1535 & $15.74 \pm 0.11$ & $13.66 \pm 0.03$ & 40.13 & 10.00 & MaxBCG,AMF,Geach,WHL12 \\
\hline 213 & 23.7849 & -1.1513 & 0.1649 & 0.1555 & $15.73 \pm 0.08$ & $13.78 \pm 0.03$ & 44.98 & 10.75 & CE,GMBCG,Geach,WHL12 \\
\hline 214 & 23.8864 & 0.3775 & 0.1486 & 0.1523 & $15.66 \pm 0.10$ & $13.77 \pm 0.03$ & 34.93 & 9.42 & Geach \\
\hline 215 & 23.9820 & 0.5584 & 0.1160 & 0.1351 & $15.33 \pm 0.07$ & $13.58 \pm 0.03$ & 33.28 & 11.50 & New \\
\hline 216 & 24.3334 & 0.9561 & 0.1780 & 0.1976 & $15.85 \pm 0.09$ & $13.74 \pm 0.03$ & 46.46 & 10.08 & New \\
\hline 217 & 24.7484 & -0.8523 & 0.1202 & 0.1179 & $15.53 \pm 0.09$ & $13.61 \pm 0.03$ & 44.34 & 14.83 & New \\
\hline 218 & 24.8218 & 0.3343 & 0.2212 & 0.1966 & $15.97 \pm 0.12$ & $13.89 \pm 0.03$ & 36.21 & 5.75 & New \\
\hline 219 & 25.2334 & 0.1560 & 0.1758 & 0.1677 & $15.58 \pm 0.09$ & $13.54 \pm 0.03$ & 51.02 & 11.25 & CE,Geach,WHL12 \\
\hline 220 & 25.3794 & -0.9283 & 0.1596 & 0.1547 & $15.60 \pm 0.08$ & $13.86 \pm 0.03$ & 36.89 & 9.17 & CE,Geach \\
\hline 221 & 25.4900 & -1.1074 & 0.1653 & 0.1560 & $15.20 \pm 0.09$ & $13.15 \pm 0.02$ & 46.18 & 11.00 & CE,MaxBCG,AMF,Geach,WHL12 \\
\hline 222 & 25.6666 & 0.1151 & 0.1120 & 0.1010 & $15.44 \pm 0.06$ & $13.77 \pm 0.03$ & 33.44 & 11.92 & New \\
\hline 223 & 25.6991 & 0.8671 & 0.1118 & 0.1015 & $15.61 \pm 0.09$ & $13.52 \pm 0.03$ & 31.76 & 11.33 & New \\
\hline 224 & 25.7110 & 0.7474 & 0.2027 & 0.1962 & $15.81 \pm 0.10$ & $13.91 \pm 0.03$ & 35.41 & 6.42 & CE,WHL12 \\
\hline 225 & 26.2515 & -0.8150 & 0.2335 & 0.1968 & $15.95 \pm 0.10$ & $13.93 \pm 0.03$ & 50.36 & 7.33 & CE,WHL12 \\
\hline 226 & 26.3010 & -0.0931 & 0.2044 & 0.1989 & $15.48 \pm 0.08$ & $13.41 \pm 0.03$ & 34.91 & 6.25 & CE,GMBCG,Geach,WHL12 \\
\hline 227 & 26.6881 & -0.6752 & 0.1002 & 0.0823 & $15.51 \pm 0.08$ & $13.65 \pm 0.03$ & 44.83 & 17.50 & AMF,WHL12 \\
\hline 228 & 27.0689 & 0.3583 & 0.1826 & 0.2061 & $\ldots$ & $13.92 \pm 0.03$ & 50.05 & 10.50 & $\mathrm{CE}$ \\
\hline 229 & 27.7459 & -1.0235 & 0.1752 & 0.1583 & $15.93 \pm 0.10$ & $13.85 \pm 0.03$ & 36.90 & 8.17 & Geach \\
\hline 230 & 27.8014 & -0.9955 & 0.2466 & 0.2428 & $16.07 \pm 0.09$ & $13.74 \pm 0.03$ & 57.76 & 7.67 & CE,WHL12 \\
\hline 231 & 28.1525 & -0.2279 & 0.1746 & 0.1768 & $15.39 \pm 0.08$ & $13.43 \pm 0.02$ & 32.96 & 7.33 & New \\
\hline 232 & 28.1750 & 1.0072 & 0.2132 & 0.2297 & $16.07 \pm 0.15$ & $13.82 \pm 0.04$ & 67.37 & 11.33 & Abell,CE,MaxBCG,GMBCG,AMF,Geach,WHL12 \\
\hline 233 & 28.1897 & -0.3932 & 0.1534 & 0.1770 & $15.68 \pm 0.09$ & $13.81 \pm 0.03$ & 43.52 & 11.33 & New \\
\hline 234 & 28.2245 & -0.8960 & 0.1141 & 0.1192 & $15.17 \pm 0.09$ & $13.27 \pm 0.03$ & 50.87 & 17.83 & New \\
\hline 235 & 28.2534 & -0.5705 & 0.1317 & 0.1331 & $16.04 \pm 0.12$ & $13.87 \pm 0.03$ & 71.54 & 21.92 & CE,MaxBCG,WHL12 \\
\hline 236 & 28.3570 & -1.1600 & 0.2138 & 0.2416 & $15.85 \pm 0.10$ & $13.59 \pm 0.03$ & 158.35 & 26.50 & Abell,CE,MaxBCG,GMBCG,AMF,Geach,WHL12 \\
\hline 237 & 29.1181 & 1.0605 & 0.1719 & 0.1826 & $15.64 \pm 0.08$ & $13.70 \pm 0.03$ & 140.27 & 31.83 & New \\
\hline 238 & 29.3485 & 0.4178 & 0.1462 & 0.1350 & $15.07 \pm 0.07$ & $13.18 \pm 0.02$ & 32.15 & 8.83 & CE,MaxBCG,GMBCG,Geach,WHL12 \\
\hline 239 & 29.4308 & -0.1504 & 0.1246 & 0.1349 & $15.85 \pm 0.10$ & $13.95 \pm 0.04$ & 38.89 & 12.58 & MaxBCG,GMBCG,Geach,WHL12 \\
\hline 240 & 29.4772 & -0.6338 & 0.1914 & 0.1884 & $15.97 \pm 0.08$ & $13.95 \pm 0.03$ & 91.52 & 18.00 & Abell,CE,GMBCG,AMF,Geach,WHL12 \\
\hline 241 & 29.4970 & -0.7244 & 0.2162 & 0.1865 & $15.78 \pm 0.09$ & $13.72 \pm 0.03$ & 86.56 & 14.25 & Abell,CE,MaxBCG,GMBCG,AMF,Geach,WHL12 \\
\hline 242 & 29.7756 & 0.8355 & 0.1443 & 0.1354 & $15.58 \pm 0.08$ & $13.73 \pm 0.03$ & 32.01 & 8.92 & Geach,WHL12 \\
\hline 243 & 29.8175 & -0.1096 & 0.1626 & 0.1550 & $15.61 \pm 0.07$ & $13.84 \pm 0.03$ & 46.98 & 11.42 & CE,MaxBCG,Geach,WHL12 \\
\hline 244 & 30.0376 & -0.8842 & 0.2128 & 0.2089 & $16.18 \pm 0.10$ & $13.69 \pm 0.03$ & 38.55 & 6.50 & WHL12 \\
\hline 245 & 30.3347 & -0.4569 & 0.1619 & 0.1597 & $15.45 \pm 0.08$ & $13.47 \pm 0.02$ & 33.07 & 8.08 & CE,MaxBCG,GMBCG,AMF,Geach,WHL12 \\
\hline 246 & 30.5012 & -0.4818 & 0.1460 & 0.1589 & $15.71 \pm 0.09$ & $13.78 \pm 0.03$ & 40.60 & 11.17 & Geach \\
\hline 247 & 31.0914 & -0.8329 & 0.1526 & 0.1367 & $15.54 \pm 0.08$ & $13.63 \pm 0.03$ & 30.24 & 7.92 & New \\
\hline 248 & 31.1257 & 0.3046 & 0.2324 & 0.1726 & $16.25 \pm 0.15$ & $13.75 \pm 0.03$ & 64.19 & 9.42 & Abell,CE,MaxBCG,AMF,Geach,WHL12 \\
\hline 249 & 31.1304 & 0.2282 & 0.1490 & 0.1631 & $16.26 \pm 0.15$ & $13.88 \pm 0.04$ & 33.76 & 9.08 & CE,MaxBCG,Geach,WHL12 \\
\hline 250 & 31.1345 & -1.0540 & 0.1699 & 0.1617 & $16.23 \pm 0.14$ & $13.82 \pm 0.03$ & 43.44 & 10.00 & CE,Geach \\
\hline 251 & 31.3167 & 0.0574 & 0.1331 & 0.1132 & $15.75 \pm 0.09$ & $13.88 \pm 0.03$ & 49.47 & 15.00 & New \\
\hline 252 & 31.3794 & 0.1897 & 0.1826 & 0.1716 & $15.37 \pm 0.09$ & $13.35 \pm 0.02$ & 30.17 & 6.33 & CE,MaxBCG,AMF,Geach,WHL12 \\
\hline 253 & 31.4742 & 0.0331 & 0.1821 & 0.1735 & $15.42 \pm 0.07$ & $13.30 \pm 0.03$ & 63.33 & 13.33 & Geach,WHL12 \\
\hline 254 & 32.4464 & -0.1118 & 0.1639 & 0.1521 & $15.54 \pm 0.09$ & $13.52 \pm 0.03$ & 36.70 & 8.83 & CE,Geach,WHL12 \\
\hline 255 & 32.5758 & -1.0184 & 0.1842 & 0.1709 & $15.66 \pm 0.08$ & $13.75 \pm 0.03$ & 79.58 & 16.50 & Abell,CE,MaxBCG,AMF,Geach,WHL12 \\
\hline 256 & 32.7266 & -1.1567 & 0.1432 & 0.1760 & $15.86 \pm 0.15$ & $13.68 \pm 0.03$ & 52.52 & 14.75 & CE,Geach,WHL12 \\
\hline 257 & 32.8846 & 0.1167 & 0.2268 & 0.2122 & $16.57 \pm 0.14$ & $14.33 \pm 0.03$ & 40.96 & 6.25 & CE,MaxBCG,GMBCG,Geach,WHL12 \\
\hline 258 & 33.0959 & -0.4210 & 0.1177 & 0.1015 & $15.37 \pm 0.08$ & $13.39 \pm 0.02$ & 32.49 & 11.08 & Geach \\
\hline 259 & 33.1749 & 0.4748 & 0.2069 & 0.2015 & $15.91 \pm 0.11$ & $13.84 \pm 0.03$ & 58.30 & 10.25 & CE,MaxBCG,AMF,Geach,WHL12 \\
\hline 260 & 33.4638 & 0.4673 & 0.1940 & 0.1820 & $15.79 \pm 0.11$ & $13.66 \pm 0.03$ & 44.43 & 8.58 & CE,MaxBCG,GMBCG,AMF,Geach,WHL12 \\
\hline
\end{tabular}


TABLE 1

CONTINUED

\begin{tabular}{|c|c|c|c|c|c|c|c|c|c|}
\hline ID & $\begin{array}{l}\text { R.A. } \\
\text { (deg) } \\
(2)\end{array}$ & $\begin{array}{c}\text { Decl. } \\
\text { (deg) } \\
\text { (3) }\end{array}$ & $z_{p}$ & $z_{s}$ & $\begin{array}{c}J \\
(\mathrm{mag}) \\
(6)\end{array}$ & $\begin{array}{c}W 1 \\
(\mathrm{mag}) \\
(7)\end{array}$ & $\begin{array}{c}R_{\text {scal }} \\
\text { (8) }\end{array}$ & $R_{m e a}$ & Note \\
\hline 261 & 33.4849 & 0.5268 & 0.2567 & 0.2126 & $15.91 \pm 0.10$ & $13.53 \pm 0.03$ & 94.30 & 11.67 & CE,MaxBCG,GMBCG,AMF,Geach,WHL12 \\
\hline 262 & 33.5527 & -0.1887 & 0.1381 & 0.1408 & $15.59 \pm 0.11$ & $13.69 \pm 0.04$ & 57.35 & 16.75 & CE,MaxBCG,GMBCG,AMF,Geach,WHL12 \\
\hline 263 & 33.7983 & 1.0014 & 0.1095 & 0.1223 & $15.18 \pm 0.08$ & $13.23 \pm 0.02$ & 43.37 & 15.75 & CE \\
\hline 264 & 33.7986 & 0.9053 & 1719 & 0.1219 & $5.96 \pm 0.09$ & $14.05 \pm 0.03$ & 74.92 & 17.00 & New \\
\hline 265 & 34.6748 & 0.1138 & 0.2509 & 0.2719 & $16.30 \pm 0.16$ & $13.66 \pm 0.03$ & 71.14 & 9.17 & CE,MaxBCG,GMBCG,AMF,Geach,WHL12 \\
\hline 266 & 36.3090 & -1.0862 & 0.1841 & 0.1694 & $15.70 \pm 0.10$ & $13.83 \pm 0.03$ & 31.32 & 6.50 & CE,GMBCG,Geach,WHL12 \\
\hline 267 & 36.7314 & -1.0641 & 227 & 967 & $5.63 \pm 0.10$ & $13.70 \pm 0.03$ & 47.97 & 15.75 & New \\
\hline 268 & 37.3373 & 0.5937 & 0.1108 & 316 & $5.12 \pm 0$ & $13.20 \pm 0.03$ & 51.22 & 18.42 & WHL12 \\
\hline 269 & 37.7454 & 1.1128 & 0.1787 & 495 & $.61 \pm 0$ & $13.52 \pm 0.03$ & 49.79 & 10.75 & $\mathrm{CE}$ \\
\hline 270 & 38.4721 & 0.0777 & 1856 & 857 & $30 \pm 0$ & $13.43 \pm 0.04$ & 42.63 & 8.75 & CE,Geach,WHL12 \\
\hline 271 & 40.2131 & -0.9324 & 0.2561 & 0.2396 & $1 \pm 0$ & $13.78 \pm 0$ & 40.21 & 5.0 & CE,MaxBCG,AMF, \\
\hline 272 & 40.6842 & -0.9660 & 1255 & 0.1797 & $4 \pm 0.09$ & $13.36 \pm 0$ & 31.91 & 10.25 & MaxBCG \\
\hline 273 & 40.8013 & -1.0201 & 5595 & 386 & $6 \pm 0$ & $13.67 \pm 0$ & 90.60 & 11.00 & CE,GMBCG,AMF,Geach,WHL12 \\
\hline 274 & 41.4178 & -0.7229 & 143 & & \pm 0 & $4 \pm 0$ & 51.98 & 8.67 & CE,MaxBCG,GMBCG, \\
\hline 275 & 41.8485 & 0.1005 & 1944 & & \pm 0 & $9 \pm 0$ & 35.49 & 6.8 & Geach,WHL12 \\
\hline 276 & 42.3182 & -0.7739 & 1493 & 376 & $3 \pm 0$ & $8 \pm 0$ & 45.02 & 12.08 & WHL12 \\
\hline 277 & 42.3603 & 0.0250 & 1821 & & \pm 0 & $4 \pm 0$ & 35.63 & 7.50 & CE,MaxBCG,GMBCG,AMF,Geach,WHL12 \\
\hline 278 & 42.4043 & 0.2698 & & & \pm 0 & $9 \pm 0$ & 30.91 & & New \\
\hline 279 & 42.9448 & -0.1646 & & & \pm 0 & $6 \pm 0$ & 34.69 & & Geach,W \\
\hline 280 & 43.1920 & 1.0847 & & & \pm 0 & $7 \pm 0$ & 81.28 & & CE,Geach,WHL \\
\hline 281 & 44.7325 & 0.2605 & & & \pm & $8 \pm 0$ & 51.21 & & WHL12 \\
\hline 282 & 44.8857 & 0.2318 & & & \pm 0 & $7 \pm 0$ & 46.93 & & $\mathrm{CE}, \mathrm{MaxBCG}, \mathrm{GMBCG}$, \\
\hline 283 & 45.6562 & -0.6689 & & & \pm & $1 \pm 0$ & 39.23 & & \\
\hline 284 & 45.7460 & -0.1935 & & & \pm & $0 \pm 0$ & 61.70 & & CE,MaxBCG,AMF,G \\
\hline 285 & 46.0997 & 0.8304 & & & $2 \pm 0$ & $13.78 \pm 0$ & 32.91 & & New \\
\hline 286 & 46.1983 & -0.9336 & & & $2 \pm 0$ & $13.84 \pm 0$ & 32.73 & & \\
\hline 287 & 46.6213 & 0.9996 & & & \pm & $4 \pm 0$ & 30.75 & & \\
\hline 288 & 47.2143 & & & & \pm & $5 \pm$ & 31.57 & & \\
\hline 289 & 48.3834 & & & & & $8 \pm 0$ & 34.66 & & \\
\hline 290 & 48.5910 & & & & 8 & $5 \pm$ & 67.06 & & MaxBCG,AMF \\
\hline 291 & 48.6184 & 0.25 & & & 1 & \pm & 46 & & $\mathrm{CE}, \mathrm{Ge}$ \\
\hline 292 & & & & & & 13 & & & \\
\hline 293 & 49.7007 & 0.6802 & & & 1( & 14 & 35.21 & & \\
\hline 294 & 50.1673 & -1.2419 & & & & 13 & 30 & & $\mathrm{CE}, \mathrm{G}$ \\
\hline 295 & 50.5087 & & & & & 13 & 32 & & \\
\hline 296 & 50.7242 & & & & 1. & 14 & & & \\
\hline 297 & 52.9716 & -0.7835 & & & 15 . & 13 & 43.00 & & CE,GMBCG,A \\
\hline 298 & 53.5507 & 1.1746 & & & 1 & $2 \pm 0$ & 49.72 & & \\
\hline 299 & 53.6419 & -1.1646 & & & 15 . & 13 & 73.76 & & CE,GMBCG, \\
\hline 300 & 54.9725 & -0.2834 & 0.1392 & & $15.44 \pm($ & 13 & 43.17 & 12.50 & \\
\hline 301 & 55.6778 & -0.2856 & 0.2795 & & $16.60 \pm($ & $46 \pm 0$ & 58.30 & 6.17 & CE,MaxBCG,GMBCG,AMF,Geach,WHL12 \\
\hline 302 & 58.2873 & -0.8459 & 0.1212 & 0.1319 & $15.59 \pm 0.09$ & $13.77 \pm 0.03$ & 34.11 & 11.33 & WHL12 \\
\hline
\end{tabular}

Note. Column(1): the sequence number; Column(2): R.A. (J2000) of cluster center in degree; Column(3): Decl. (J2000) of cluster center in degree; Column(4): photometric redshift of cluster with a typical uncertainty of 0.022 ; Column(5): spectroscopic redshift of central galaxy with a typical uncertainty of $2 \times 10^{-4}$; Column(6): $J$-band magnitude of centeral galaxy; Column(7): $W 1$-band magnitude of centeral galaxy; Column(8): scaled richness of cluster; Column(9): measured richness of cluster. Column(10): Notes for known clusters in other catalogs: Abell (Abell 1958; Abell et al. 1989); CE (Goto et al. 2002); MaxBCG (Koester et al. 2007); GMBCG (Hao et al. 2010); AMF (Szabo et al. 2011); Geach (Geach et al. 2011), WHL12 (Wen et al. 2012); newly identified clusters are labelled "New". 